Comm. Nonlin. Sci Numer. Simul. 13, (2008), 1256-1263. 


\title{
Discrepancy principle for DSM II
}

\author{
A.G. Ramm \\ Mathematics Department, Kansas State University, \\ Manhattan, KS 66506-2602, USA \\ ramm@math.ksu.edu
}

\begin{abstract}
Let $A y=f, A$ is a linear operator in a Hilbert space $H, y \perp N(A):=\{u$ : $A u=0\}, R(A):=\{h: h=A u, u \in D(A)\}$ is not closed, $\left\|f_{\delta}-f\right\| \leq \delta$. Given $f_{\delta}$, one wants to construct $u_{\delta}$ such that $\lim _{\delta \rightarrow 0}\left\|u_{\delta}-y\right\|=0$. Two versions of discrepancy principles for the DSM (dynamical systems method) for finding the stopping time and calculating the stable solution $u_{\delta}$ to the original equation $A y=f$ are formulated and mathematically justified.
\end{abstract}

\section{Introduction.}

Let $A$ be a linear bounded operator in a Hilbert space $H$ (or in a Banach space $X$ ), and equation

$$
A u=f
$$

be solvable, possibly non-uniquely. Let $N(A)=N$ and $R(A)$ denote the null-space and the range of $A$, respectively. Denote by $y$ the (unique) minimal-norm solution to (1), $y \perp N$. Given $f_{\delta},\left\|f_{\delta}-f\right\| \leq \delta$, one wants to find a stable approximation $u_{\delta}$ to $y$ :

$$
\lim _{\delta \rightarrow 0}\left\|u_{\delta}-y\right\|=0
$$

There are many ways to do this: variational regularization, quasisolutions, iterative regularization (see, e.g., [1], [3]).

In [4] a version of the discrepancy principle for DSM was proved. This version consisted in solving the equation for $t$ :

$$
\left\|T_{a(t)}^{-1} A^{*} f_{\delta}-f_{\delta}\right\|=c \delta
$$

MSC: 47A52, 47D06, 47N40, 65L08, 65L09, 65L20; PACS: 0.2.30.+g, 0.2.60.+y, 0.2.70.+d,

Key words: dynamical systems method (DSM), ill-posed problems, discrepancy principle, evolution equation, spectral theory 
where $c=$ const $\in(1,2)$, and $a(t)>0$ was monotonically decaying and satisfied the assumption:

$$
\lim _{t \rightarrow \infty} \sup _{\frac{t}{2} \leq s \leq t}|\dot{a}(s)| a^{-2}(t)=0 .
$$

Here we relax the assumptions on $a(t)$ and make the principle easier to apply numerically.

We study a new version of the dynamical systems method (DSM) for finding $u_{\delta}$ :

$$
\dot{u}_{\delta}(t)=-u_{\delta}(t)+T_{a(t)}^{-1} A^{*} f_{\delta}, \quad u_{\delta}(0)=u_{0},
$$

where $T:=A^{*} A$ is selfadjoint, $T_{a}:=T+a I, I$ is the identity operator,

$$
0<a(t) ; \quad a(t) \searrow 0 \text { as } t \rightarrow \infty ; \quad \lim _{t \rightarrow \infty} \frac{\dot{a}}{a}=0, \quad \dot{a}:=\frac{d a}{d t} .
$$

The element $u_{\delta}$ in $(2)$ is $u_{\delta}\left(t_{\delta}\right)$, where $u_{\delta}(t)$ is the solution to (3), and $t_{\delta}$, the stopping time, is found from the following equation for the unknown $t$ :

$$
\int_{0}^{t} e^{-(t-s)} a(s)\left\|Q_{a(s)}^{-1} f_{\delta}\right\| d s=c \delta, \quad c \in(1,2),
$$

where $Q:=A A^{*}$ is selfadjoint, $Q_{a}=Q+a I, c$ is a constant, and $\left\|f_{\delta}\right\|>c \delta$. This equation we call a discrepancy principle. About other versions of discrepancy principles see [2], [3]-[8].

The main result of this paper is the following theorem.

Theorem 1. Assume that (4) holds, $\left\|f_{\delta}\right\|>c \delta$, and

$$
\lim _{t \rightarrow \infty} e^{t} a(t)\left\|Q_{a(t)}^{-1} f_{\delta}\right\|=\infty .
$$

Then equation (5) has a unique solution $t_{\delta}$,

$$
\lim _{\delta \rightarrow 0} t_{\delta}=\infty
$$

and (2) holds with $u_{\delta}:=u_{\delta}\left(t_{\delta}\right)$.

Remark 1. Assumption (6) is always satisfied if $f_{\delta} \notin R(A)$. Indeed,

$$
\lim _{a \rightarrow 0}\left\|a Q_{a}^{-1} f_{\delta}\right\|^{2}=\lim _{a \rightarrow 0} \int_{0}^{\|Q\|} \frac{a^{2} d\left(E_{\lambda} f_{\delta}, f_{\delta}\right)}{(a+\lambda)^{2}}=\left\|P f_{\delta}\right\|^{2}>0,
$$

where $E_{\lambda}$ is the resolution of the identity, corresponding to the selfadjoint operator $Q, P$ is the orthoprojector onto the null space $N(Q)$ of $Q, N(Q)=N\left(A A^{*}\right)=N\left(A^{*}\right):=N^{*}$, and $\left\|P_{N^{*}} f_{\delta}\right\|>0$ if $f_{\delta} \notin R(A)$, because $\overline{R(A)}=\left(N^{*}\right)^{\perp}$.

In Section 2 we prove Theorem 1 and Theorem 2, which says that (2) holds without assumption (6) but with an extra assumption $\lim _{t \rightarrow \infty} \frac{\dot{a}(t)}{a^{2}(t)}=0$. 


\section{Proofs.}

Let

$$
h(t):=a(t)\left\|A_{a(t)}^{-1} f_{\delta}\right\|:=a(t) g(t) .
$$

Lemma 1. Assume (6). Then

$$
\lim _{t \rightarrow \infty} \frac{e^{t} h(t)}{\int_{0}^{t} e^{s} h(s) d s}=1
$$

provided that

$$
\lim _{t \rightarrow \infty} \frac{\dot{h}}{h}=0 .
$$

Proof of Lemma 1. Apply L'Hospital's rule to (8) and conclude that (8) holds if

$$
\lim _{t \rightarrow \infty} \frac{e^{t} h(t)+e^{t} \dot{h}}{e^{t} h(t)}=1
$$

Relation (10) holds if (9) holds.

Lemma 1 is proved.

Lemma 2. Relation (9) holds if and only if

$$
\lim _{t \rightarrow \infty} \frac{\left(h^{2}\right)^{\bullet}}{h^{2}}=0, \quad\left(h^{2}\right)^{\bullet}:=\frac{d\left(h^{2}\right)}{d t} .
$$

Proof of Lemma 2. We have

$$
\lim _{t \rightarrow \infty} \frac{\left(h^{2}\right)^{\bullet}}{h^{2}}=2 \lim _{t \rightarrow \infty} \frac{\dot{h}}{h}
$$

Lemma 2 is proved.

We have $h=a g$. Therefore

$$
\frac{\left(h^{2}\right)^{\bullet}}{h^{2}}=\frac{\left(a^{2}\right)^{\bullet} g^{2}+a^{2}\left(g^{2}\right)^{\bullet}}{a^{2} g^{2}}=\frac{\left(a^{2}\right)^{\bullet}}{a^{2}}+\frac{\left(g^{2}\right)^{\bullet}}{g^{2}} .
$$

If (4) holds, then Lemma 2 implies

$$
\lim _{t \rightarrow \infty} \frac{\left(a^{2}\right)^{\bullet}}{a^{2}}=0 .
$$

Let us prove that

$$
\lim _{t \rightarrow \infty} \frac{\left(g^{2}\right)^{\bullet}}{g^{2}}=0 .
$$


Let $E_{s}$ be the resolution of the identity of the selfadjoint operator $Q$. Then

$$
g^{2}(t)=\int_{0}^{b} \frac{d \rho(s)}{[s+a(t)]^{2}}, \quad \rho(s):=\left(E_{s} f_{\delta}, f_{\delta}\right), \quad b:=\|Q\|,
$$

and

$$
\begin{aligned}
\left(g^{2}\right)^{\bullet}= & -2 \dot{a} \int_{0}^{b} \frac{d \rho(s)}{[s+a(t)]^{3}}=2 \frac{|\dot{a}|}{a} \int_{0}^{b} \frac{a(t) d \rho(s)}{[s+a(t)]^{3}} \\
& \leq 2 \frac{|\dot{a}(t)|}{a(t)} \int_{0}^{b} \frac{d \rho(s)}{[s+a(t)]^{2}}=\frac{2|\dot{a}(t)|}{a(t)} g^{2}(t),
\end{aligned}
$$

where we have used the monotone decay of $a(t)$, which implies $-\dot{a}=|\dot{a}|$. Our results remain valid for $b=\infty$ and their proofs are essentially the same.

From (4), (16) and (17) it follows that

$$
0 \leq \lim _{t \rightarrow \infty} \frac{\left(g^{2}\right)^{\bullet}}{g^{2}} \leq 2 \lim _{t \rightarrow \infty} \frac{|\dot{a}|}{a}=0
$$

We have proved the following lemma.

Lemma 3. If (4) holds, then

$$
\lim _{t \rightarrow \infty} \frac{\left(h^{2}(t)\right)^{\bullet}}{h^{2}(t)}=0
$$

From Lemmas 1-3 we obtain the following result.

Lemma 4. If (4) and (6) hold, then

$$
\lim _{t \rightarrow \infty} \frac{\int_{0}^{t} e^{-(t-s)} h(s) d s}{h(t)}=1 .
$$

Proof of Lemma 4. Relation (20) is equivalent to

$$
\lim _{t \rightarrow \infty} \frac{e^{t} h(t)}{\int_{0}^{t} e^{s} h(s) d s}=1 .
$$

By Lemmas 1-3, relation (21) holds if

$$
\lim _{t \rightarrow \infty} \frac{\dot{a}}{a}=0
$$

This relation holds by assumption (4).

Lemma 4 is proved.

Now let us prove Theorem 1. 
Proof of Theorem 1. By Lemma 4 equation (5) for large $t$ can be written as

$$
a(t)\left\|Q_{a(t)}^{-1} f_{\delta}\right\|=c \delta[1+o(1)], \quad t \rightarrow \infty .
$$

Denote $c[1+o(1)]$ by $c_{1}$. If $c \in(1,2)$, then $c_{1} \in(1,2)$ for sufficiently large $t$. Fix a sufficiently large $t$ and denote $a(t):=a$. Consider the equation

$$
\psi(a):=a^{2}\left\|Q_{a}^{-1} f_{\delta}\right\|^{2}=\int_{0}^{b} \frac{a^{2} d \rho(s)}{(a+s)^{2}}=c_{1}^{2} \delta^{2} .
$$

The function $\psi(a)$ is defined on $(0, \infty)$, is monotonically growing and continuous,

$$
\psi(+\infty)=\int_{0}^{b} d \rho=\left\|f_{\delta}\right\|^{2}>c_{1}^{2} \delta^{2}, \quad \psi(+0)=\left\|P f_{\delta}\right\|^{2},
$$

where $P, P:=E_{0}-E_{0-}$, is the orthogonal projection onto the null-space $N(Q)$ of the operator $Q, N(Q)=N\left(A A^{*}\right)=N\left(A^{*}\right):=N^{*}$. Since $\left\|f_{\delta}-f\right\| \leq \delta$, and $f \in R(A) \perp$ $N\left(A^{*}\right)$, we have

$$
\left\|P f_{\delta}\right\| \leq\left\|P\left(f_{\delta}-f\right)\right\|+\|P f\| \leq \delta
$$

because $\|P\| \leq 1$ and $\|P f\|=0$. Therefore equation (24) has a unique solution $a=a_{\delta}$, $\lim _{\delta \rightarrow 0} a_{\delta}=0$. Consequently, equation (5) has a unique solution $t_{\delta}$, which can be found from the equation

$$
a(t)=a_{\delta}
$$

Since $a(t) \searrow 0$, we have

$$
\lim _{\delta \rightarrow 0} t_{\delta}=\infty
$$

Finally, let us prove that the element $u_{\delta}:=u_{\delta}\left(t_{\delta}\right)$ satisfies relation (2). First we give a simple proof of this relation under an additional assumption (39), see below. Then we give a more complicated proof which does not use any additional assumptions.

We have

$$
u_{\delta}(t)=e^{-t} u_{0}+\int_{0}^{t} e^{-(t-s)} T_{a(s)}^{-1} A^{*} f_{\delta} d s .
$$

Thus

$$
\begin{aligned}
\left\|u_{\delta}(t)-y\right\| \leq e^{-t}\left\|u_{0}\right\| & +\left\|\int_{0}^{t} e^{-(t-s)} T_{a(s)}^{-1} A^{*}\left(f_{\delta}-f\right) d s\right\| \\
& +\left\|\int_{0}^{t} e^{-(t-s)} T_{a(s)}^{-1} A^{*} f d s-y\right\|:=j_{1}+j_{2}+j_{3} .
\end{aligned}
$$

Clearly, $\lim _{t \rightarrow \infty} j_{1}=0$. We have $\left\|T_{a}^{-1} A^{*}\right\| \leq \frac{1}{2 \sqrt{a}}, a>0$.

Indeed, $T^{-1} A^{*}=A^{*} Q_{a}^{-1}=U Q^{\frac{1}{2}} Q_{a}^{-1}$, where $U$ is a partial isometry, and we have used the formula $T_{a}^{-1} A^{*}=A^{*} Q_{a}^{-1}$ and the polar decomposition $A^{*}=U Q^{\frac{1}{2}}$. Thus,

$$
\left\|T^{-1} A^{*}\right\| \leq\left\|Q^{\frac{1}{2}} Q_{a}^{-1}\right\|=\sup _{\delta>0} \frac{s^{\frac{1}{2}}}{s+a}=\frac{1}{2 \sqrt{a}} .
$$


Consequently,

$$
j_{2} \leq \frac{\delta}{2 \sqrt{a(t)}}
$$

Furthermore, $f=A y$, so

$$
\left\|\int_{0}^{t} e^{-(t-s)} T_{a(s)}^{-1} A^{*} A y d s-y\right\| \leq\|y\| e^{-t}+\int_{0}^{t} e^{-(t-s)} a(s)\left\|T_{a(s)}^{-1} y\right\| d s,
$$

and

$$
\lim _{a \rightarrow 0} a^{2}\left\|T_{a}^{-1} y\right\|^{2}=\lim _{a \rightarrow 0} \int_{0}^{b} \frac{a^{2} d\left(F_{s} y, y\right)}{(a+s)^{2}}=\|\mathcal{P} y\|^{2}=0,
$$

where $F_{s}$ is the resolution of the identity corresponding to the selfadjoint operator $T=$ $A^{*} A, \mathcal{P}$ is the orthogonal projection onto $N(T)=N(A)$, and $\mathcal{P} y=0$ because $y \perp N$.

It follows from (31) and (32) that

$$
\lim _{t \rightarrow \infty} j_{3}=0
$$

Indeed, if

$$
\varphi(s):=a(s)\left\|T_{a(s)}^{-1} y\right\| \rightarrow 0 \text { as } s \rightarrow \infty,
$$

then, as follows from Lemma 6 (see below), we have

$$
\lim _{t \rightarrow \infty} \int_{0}^{t} e^{-(t-s)} \varphi(s) d s=0
$$

If

$$
\lim _{\delta \rightarrow 0} \frac{\delta}{\sqrt{a\left(t_{\delta}\right)}}=0
$$

and

$$
\lim _{\delta \rightarrow 0} t_{\delta}=\infty
$$

then (29), (30) and (33) imply relation (2) with $u_{\delta}=u_{\delta}\left(t_{\delta}\right)$.

Equations (23) and (26) show that

$$
a\left\|Q_{a}^{-1} f_{\delta}\right\|=c_{1} \delta, \quad 1<c_{1}<2, \quad a:=a_{\delta} .
$$

We do not have a proof of the relation (36), and it is not known, in general, if (36) holds without additional assumptions. We can prove (36) under the following additional assumption:

$$
y=T^{\gamma} v, \quad 0<\gamma<\frac{1}{2} .
$$

Lemma 5. If (39) holds and $a=a_{\delta}$ solves (38), then (36) holds. 
Proof of Lemma 5. We have

$$
f=A y=A T^{\gamma} v
$$

and (38) implies

$$
\begin{aligned}
\frac{c_{1} \delta}{\sqrt{a_{\delta}}} & \leq \sqrt{a_{\delta}}\left(\left\|Q_{a_{\delta}}^{-1}\left(f-f_{\delta}\right)\right\|+\left\|Q_{a_{\delta}}^{-1} A T^{\gamma} v\right\|\right) \\
& \leq \sqrt{a_{\delta}}\left(\frac{\delta}{a_{\delta}}+\left\|A T_{a_{\delta}}^{-1} T^{\gamma} v\right\|\right) \leq \frac{\delta}{\sqrt{a_{\delta}}}+\sqrt{a_{\delta}}\|v\|\left\|T_{a_{\delta}}^{-1} T^{\frac{1}{2}+\gamma}\right\|
\end{aligned}
$$

where we have used the obvious estimate $\left\|Q_{a}^{-1}\right\| \leq \frac{1}{a}$, which holds for any $a>0$, and the commutation relation

$$
Q_{a}^{-1} A=A T_{a}^{-1}
$$

Therefore

$$
\left(c_{1}-1\right) \frac{\delta}{\sqrt{a_{\delta}}} \leq \sqrt{a_{\delta}}\|v\| \max _{s>0} \frac{s^{\frac{1}{2}+\gamma}}{s+a_{\delta}}=c\|v\| a_{\delta}^{\gamma} \rightarrow 0 \text { as } \delta \rightarrow 0,
$$

where $c=c(\gamma)>0$ is a constant, $c_{1}>1$,

$$
\max _{s>0} \frac{s^{\frac{1}{2}+\gamma}}{s+a_{\delta}}=c(\gamma) a^{\gamma-\frac{1}{2}}
$$

and

$$
c=c(\gamma)=p^{p}(1-p)^{1-p}
$$

with $p:=\gamma+\frac{1}{2}$.

Lemma 5 is proved.

However, we want to prove relation (2) with $u_{\delta}=u_{\delta}\left(t_{\delta}\right)$ (where $t_{\delta}$ solves $(26)$, and $a_{\delta}$ solves (38)) without any extra assumptions of the type (39).

To accomplish this, we propose the following argument.

Equation (38) can be written as

$$
c_{1} \delta=\left\|a Q_{a}^{-1} f_{\delta}\right\|=\left\|(a+Q-Q) Q_{a}^{-1} f_{\delta}\right\|=\left\|Q Q_{a}^{-1} f_{\delta}-f_{\delta}\right\|=\left\|A T_{a}^{-1} A^{*} f_{\delta}-f_{\delta}\right\| .
$$

Let us denote

$$
w_{\delta}:=T_{a_{\delta}}^{-1} A^{*} f_{\delta},
$$

where $a_{\delta}$ is the solution to equation (42). It is proved in [3, p.22] that

$$
\lim _{\delta \rightarrow 0}\left\|w_{\delta}-y\right\|=0
$$

By formula (28) the relation

$$
\lim _{\delta \rightarrow 0} u_{\delta}\left(t_{\delta}\right)=y
$$

holds due to the following lemma. 
Lemma 6. If

$$
\lim _{t \rightarrow \infty} q(t)=q
$$

then

$$
\lim _{t \rightarrow \infty} \int_{0}^{t} e^{-(t-s)} q(s) d s=q .
$$

Proof of Lemma 6. Take $\varepsilon>0$ arbitrarily small. Using (45), find $t(\varepsilon)$ such that

$$
|q(t)-q| \leq \frac{\varepsilon}{4} \quad \text { for } \quad t \geq t(\varepsilon)
$$

Then

$$
I:=\left|\int_{0}^{t} e^{-(t-s)} q(s) d s-q\right| \leq\left|\int_{0}^{t(\varepsilon)} e^{-(t-s)} q(s) d s\right|+\left|\int_{t(\varepsilon)}^{t} e^{-(t-s)} q(s) d s-q\right|:=I_{1}+I_{2} .
$$

Take $\tau(\varepsilon)$ sufficiently large, so that

$$
I_{1}<\frac{\varepsilon}{2} \quad \text { for } \quad t>\tau(\varepsilon)
$$

and

$$
\begin{aligned}
I_{2} & \leq \int_{t(\varepsilon)}^{t} e^{-(t-s)}|q(s)-q| d s+\left|\int_{t(\varepsilon)}^{t} e^{-(t-s)} d s q-q\right| \\
& \leq \frac{\varepsilon}{4}\left(1-e^{-(t-t(\varepsilon))}\right)+|q| e^{-(t-t(\varepsilon))}<\frac{\varepsilon}{2} \quad \text { for } \quad t>\tau(\varepsilon) .
\end{aligned}
$$

Then

$$
I \leq \varepsilon \quad \text { if } \quad t>\tau(\varepsilon)
$$

Lemma 6 is proved.

Theorem 1 is proved.

We can simplify our arguments if the following extra assumption on $a(t)$ is made in addition to (4):

$$
\lim _{t \rightarrow \infty} \frac{\dot{a}(t)}{a^{2}(t)}=0 .
$$

Theorem 2. Assume (4) and (51). Let $a_{\delta}$ solve (38) and $t_{\delta}$ solve (26). Then relation (2) holds with $u_{\delta}:=u_{\delta}\left(t_{\delta}\right)$, where $u_{\delta}(t)$ is given in (28).

Proof of Theorem 2. We have

$$
\left\|u_{\delta}\left(t_{\delta}\right)-y\right\| \leq\left\|u_{\delta}\left(t_{\delta}\right)-w_{\delta}\left(t_{\delta}\right)\right\|+\left\|w_{\delta}\left(t_{\delta}\right)-y\right\|,
$$

where $w_{\delta}(t):=T_{a(t)}^{-1} A^{*} f_{\delta}$. It is known ([3], p. 22) that

$$
\lim _{\delta \rightarrow 0}\left\|w_{\delta}\left(t_{\delta}\right)-y\right\|=0 .
$$


Let us prove that

$$
\lim _{\delta \rightarrow 0}\left\|u_{\delta}\left(t_{\delta}\right)-w\left(t_{\delta}\right)\right\|=0
$$

We have

$$
u_{\delta}(t)=e^{-t} u_{0}+\int_{0}^{t} e^{-(t-s)} w_{\delta}(s) d s
$$

and

$$
\int_{0}^{t} e^{-(t-s)} w_{\delta}(s) d s=\left.e^{-(t-s)} w_{\delta}(s)\right|_{0} ^{t}-\int_{0}^{t} e^{-(t-s)} \dot{w}_{\delta}(s) d s .
$$

From (54) and (55) we get

$$
\left\|u_{\delta}(t)-w_{\delta}(t)\right\| \leq c e^{-t}+\int_{0}^{t} e^{-(t-s)}\left\|\dot{w}_{\delta}(s)\right\| d s .
$$

From (54) and Lemma 6 it follows that

$$
\lim _{\delta \rightarrow 0}\left\|u_{\delta}\left(t_{\delta}\right)-w_{\delta}\left(t_{\delta}\right)\right\|=0
$$

provided that

$$
\lim _{t \rightarrow \infty}\left\|\dot{w}_{\delta}(t)\right\|=0 .
$$

To prove (57) we use assumption (51) and the following estimate:

$$
\left\|\dot{w}_{\delta}(t)\right\|^{2}=\int_{0}^{\|T\|} \frac{\dot{a}^{2}(t)}{[a(t)+\lambda]^{4}} d\left(F_{\lambda} A^{*} f_{\delta}, A^{*} f_{\delta}\right) \leq \frac{\dot{a}^{2}(t)}{a^{4}(t)}\left\|A^{*} f_{\delta}\right\|^{2} \underset{t \rightarrow \infty}{\longrightarrow} 0
$$

where $F_{\lambda}$ is the resolution of the identity corresponding to the operator $T$ (cf formula (32)). Theorem 2 is proved.

Remark 2. Assumption (51) holds, for example, if $a(t)=\frac{c_{0}}{\left(c_{1}+t\right)^{b}}$ for sufficiently large $t$, $t>T, c_{0}, c_{1}, b>0$ are constants, $b \in(0,1)$, and $T>0$ is an arbitrary large fixed number. On the initial finite interval $t \in[0, T]$ the function $a(t)>0$ may decay arbitrarily fast.

\section{References}

[1] Ivanov, V., Vasin, V., Tanana, V. , Theory of linear ill-posed problems, Nauka, Moscow, 1978. (In Russian).

[2] Morozov, V., Methods of solving incorrectly posed problems, Springer-Verlag, New York, 1984.

[3] Ramm, A. G., Inverse problems, Springer, New York, 2005.

[4] — Discrepancy principle for the dynamical systems method, Communic. in Nonlinear Sci. and Numer. Simulation, 10, N1, (2005), 95-101. 
[5] — Regularization of ill-posed problems with unbounded operators, J. Math. Anal. Appl., 271, (2002), 547-550.

[6] — On the discrepancy principle, Nonlinear Functional Anal. and Applic., 8, N2, (2003), 307-312.

[7] — A new discrepancy principle, J. Math. Anal. Appl., 310, (2005), 342-345.

[8] — Dynamical systems method for solving operator equations, Elsevier, Amsterdam, 2007. 